\title{
A reduced-complexity model for river delta formation - Part 2: Assessment of the flow routing scheme
}

\author{
M. Liang ${ }^{1,2}$, N. Geleynse ${ }^{2, *}$, D. A. Edmonds ${ }^{3}$, and P. Passalacqua ${ }^{1}$ \\ ${ }^{1}$ Department of Civil, Architectural and Environmental Engineering and Center for Research in Water \\ Resources, The University of Texas at Austin, Austin, Texas, USA \\ ${ }^{2}$ Department of Geological Sciences, The University of Texas at Austin, Austin, Texas, USA \\ ${ }^{3}$ Department of Geological Sciences, Indiana University, Bloomington, Indiana, USA \\ "now at: ARCADIS, Water and Environment Division, Zwolle, the Netherlands \\ Correspondence to: M. Liang (manliang@utexas.edu)
}

Received: 25 June 2014 - Published in Earth Surf. Dynam. Discuss.: 28 July 2014

Revised: 5 December 2014 - Accepted: 8 January 2015 - Published: 28 January 2015

\begin{abstract}
In a companion paper (Liang et al., 2015) we introduced a reduced-complexity model (RCM) for river delta formation, developed using a parcel-based "weighted random walk" method for routing water and sediment flux. This model (referred to as DeltaRCM) consists of a flow routing scheme as the hydrodynamic component (referred to as FlowRCM) and a set of sediment transport rules as the morphodynamic component. In this work, we assess the performance of FlowRCM via a series of hydrodynamic tests by comparing the model outputs to Delft3D and theoretical predictions. These tests are designed to reveal the capability of FlowRCM to resolve flow field features that are critical to delta dynamics at the level of channel processes. In particular, we focus on (1) backwater profile, (2) flow around a mouth bar, (3) flow through a single bifurcation, and (4) flow through a distributary channel network. We show that while the simple rules are not able to reproduce all fine-scale flow structures, FlowRCM captures flow field features that are essential to deltaic processes such as bifurcations and avulsions, the partitioning of flux between channels and inundated islands, and the instability of flux distribution at channel mouths which is responsible for mouth-bar growth. Finally, we discuss advantages and limitations of FlowRCM and identify environments most suitable for it.
\end{abstract}

\section{Introduction}

Flow routing plays a fundamental role in geomorphologic modeling. Although all models resolving coupled fluid flow and sediment transport are simplified to a certain degree, reduced-complexity models (RCMs) gain their name in comparison to more detailed reductionism models, sometimes referred to as "high-fidelity" models, typically based on rigorous computational fluid dynamics (CFD) solutions (e.g., Lane et al., 2002; Lesser et al., 2004; Nicholas, 2013; Duan and Julien, 2010; Siviglia et al., 2013). Reduced-complexity (RC) flow routing schemes are typically in the form of cellular models, in which topography is represented by a lattice of cells with elevation information and wherein flux is calculated by cellular rules abstracting governing physics (Nicholas, 2005; Murray, 2007; Paola et al., 2011). Due to the highly rule-based nature and extensive parameterization, $\mathrm{RC}$ flow routing schemes are often tailored to the characteristics of the target environment, and the processes that are simplified and represented are carefully selected. For steep terrains such as drainage networks and alluvial fans, routing schemes are typically based on topographic slopes alone. Examples include single-direction methods such as the steepest descent method (O'Callaghan and Mark, 1984), and the multidirection method (MFD) (Freeman, 1991). The successive flow-routing method (Pelletier, 2008) modifies the MFD method by accounting for the effects of flow depth on flow pathways in an iterative fashion. The method can handle divergent flow with flooding, although it is still primarily based on topographic slope. For flow routing in fluvial channels and floodplain inundation, flow depth and water surface slope 
have to be taken into account (Bates et al., 2010). Routing schemes for these environments are usually based on equations such as Manning's to convert discharge to flow depth and obtain the local water surface slope and thus the flux between neighboring cells. Models in this category include LISFLOOD-FP (Bates and de Roo, 2000; Bates et al., 2010), CAESAR (Coulthard et al., 2002; Van De Wiel et al., 2007) and the ones proposed by Parsons and Fonstad (2007), Nicholas (2010) and Larsen and Harvey (2011).

Despite these efforts, there is a relatively small number of flow routing schemes developed for river deltas, which is surprising given the importance of deltaic environments for people and resources, and their increasingly recognized vulnerability to natural and anthropogenic disturbance (Syvitski et al., 2009). Existing RC flow routing schemes for channelresolving river delta formation are the model proposed by Seybold et al. (2007) and the hydrodynamic component (referred to as FlowRCM) of the parcel-based delta formation model (DeltaRCM) developed by Liang et al. (2015). The challenges of routing flow in a deltaic environment include (i) low topographic slopes (typically $10^{-4}-10^{-5}$ ), (ii) lowFroude-number flow which exhibits strong backwater effects making it difficult to design localized rules essential for RC routing schemes, and (iii) distributary channel networks with strong spatial variation in flow directions and loops which further complicate flow routing. Although methods such as LISFLOOD-FP, CAESAR and the one proposed by Nicholas et al. (2012) have shown good performance in low-gradient environments, they would likely need to be modified to account for flow characteristics of deltaic environments (Liang et al., 2015).

In this paper we provide a comprehensive assessment of our reduced complexity flow routing model FlowRCM, testing for the first time the performance of a RC model for deltas. We see this assessment as a test of model plausibility (Hardy et al., 2003), or the credibility of the processes represented in the model, rather than a "validation" of the model as often performed in numerical modeling. Some argue that validation of numerical models in Earth science is impossible and model confirmation by the demonstration of agreement between observation and prediction is inherently partial (Oreskes et al., 1994). Still, approaches at different levels have been defined and applied (Martin and Church, 2004) to compare a simulated landscape and a real landscape in terms of (i) behavior of governing processes (e.g., Tucker and Bras, 1998), (ii) qualitative consistency (e.g., Howard, 1997), (iii) full quantitative comparison (e.g., Ferguson et al., 2001), and (iv) statistical properties (e.g., Willgoose, 1994). Validating RCMs poses difficulties for several reasons as outlined below. First of all, RCMs in general put emphasis on "explanation" rather than "prediction" (Murray, 2007). Therefore, the validation for a RCM is different in the sense that evaluation of the representation of the classical physics in full is not the subject of the validation. Rather, the validation focuses on the evaluation of the correct repre- sentation of the behavior and physical structures of the system under consideration. Due to the wide spectrum of purpose and complexity of RCMs, standards for model validation are still poorly defined. Statistical methods offer quantitative metrics for validation, but the identification of the most revealing and discriminating statistics of a certain system is a challenge in itself. Examples of on-going efforts in developing better metrics for the quantitative description of river deltas include the work by Wolinsky et al. (2010), Edmonds et al. (2011), and Passalacqua et al. (2013). Second, a large portion of RCMs are built for geological timescales, such as models for studying channel avulsions and alluvial architecture (e.g., Jerolmack and Paola, 2007; Karssenberg and Bridge, 2008). Validation of such models requires intensive stratigraphic data which are not easily available. This issue has been addressed by many researchers, calling for a combined effort of model development and field observations (Overeem et al., 2005; Hajek and Wolinsky, 2012). Third, for cellular models, model validation (whether the model correctly represents physical processes) and verification (whether the numerical solutions are in agreement with the given equations) are sometimes mixed. For example, achieving a grid-independent solution is inherently more difficult than for other types of numerical models as the grid structure represents an implicit element of the process parameterization (Nicholas, 2005). After recent improvement of $\mathrm{RC}$ modeling techniques, especially in flow routing schemes, it is possible to develop RCMs that are capable of predicting flow patterns with accuracy comparable to CFD-based models (Nicholas et al., 2012). In the validation work by Nicholas et al. (2012), an intercomparison of a relatively simple RCM with two CFD models shows that all the three models are able to replicate patterns of depth-averaged velocity and unit discharge evident in acoustic Doppler current profiler (ADCP) cross-sectional surveys.

We design a series of tests for the $\mathrm{RC}$ flow routing scheme - referred to as FlowRCM - of the delta formation model introduced in Part 1 (Liang et al., 2015). With the proposed tests we aim to examine to what extent FlowRCM is able to reproduce hydrodynamic details, in comparison to higherfidelity CFD-based models such as Delft3D, which have been shown to accurately model hydrodynamics (Lesser et al., 2004). The goal of our analysis is not to match Delft3D simulations, but to identify in retrospective similarities and differences in model results, and to identify what hydrodynamic features are the controlling factors in delta morphodynamics. The tests are designed in a way that each case represents a critical hydrodynamic process essential to delta morphology at the scale of channel dynamics: (1) backwater profile, (2) flow around a mouth bar, (3) flow through a single bifurcation, and (4) flow through a distributary channel network.

The paper is organized as follows. In Sect. 2, we briefly describe the key steps in FlowRCM, based on a parcel-based "weighted random walk" method. In Sect. 3, we present the design, setup and results of the test cases and their relevance 
A

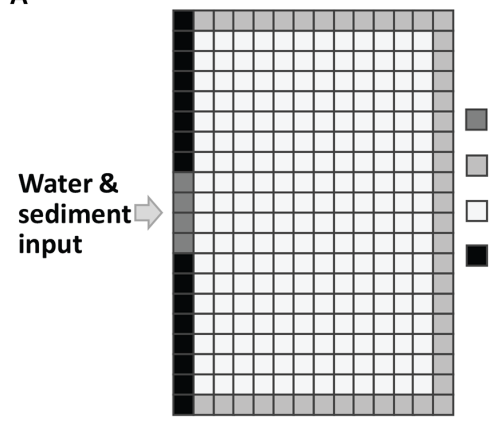

B

Inlet channel cells

Fixed water level

Basin cells

No-flow boundary

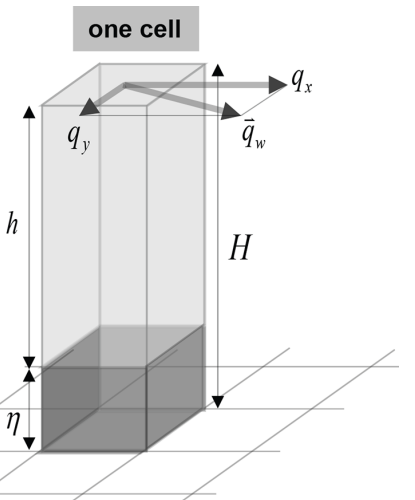

Figure 1. Basic setup of the model. (a) The calculation domain is represented by a lattice of square cells (shown as a diagram). (b) Primary values associated with each cell include flow depth, water surface elevation, bed elevation and flow unit discharge vector.

to delta formation. We discuss our results and ideas for future research in Sect. 4. Finally, we present our conclusions in Sect. 5.

\section{Review of the proposed flow routing scheme}

Here we give a brief review of our reduced-complexity flow routing scheme FlowRCM, focusing on the key steps and parameterizations (for a complete description refer to Part 1; Liang et al., 2015). In this section, we assume a nondeformable topography (i.e., no morphodynamic processes), over which the flow routing scheme resolves the depthaveraged flow field and the water surface profile given appropriate initial and boundary conditions.

The topography is represented by a lattice of square cells. At each cell, quantities such as bed elevation $\eta$, water surface elevation $\nabla H$, and water unit discharge $\nabla H$ are recorded (Fig. 1). Water depth $h$ can be calculated by taking the difference between water surface elevation and bed elevation (with $H$ and $\eta$ both relative to a reference datum). Water flux is represented by a large number of small water parcels, typically hundreds to a few thousands. With a larger number of parcels, the probability-based routing scheme is less affected by extreme events, but requires longer computational time. We found that the magnitude of 1000 works best in terms of efficiency. The solution is reached in an iterative fashion: first, parcels are routed individually from cell to cell based on a probability field, then the flow field and water surface elevation are updated. This process is repeated until the calculated flow field and water surface elevation converge. Convergence is defined as a dynamic equilibrium of flux distribution, which is typically achieved in 500-1000 iteration steps. We do not use the classic convergence criterion which is based on the difference between consecutive iterations due to two factors: (1) the probabilistic nature of the routing scheme introduces considerable noise between iterations, (2) there is an "oscillation" behavior caused by the feedback between underrelaxed water surface update and wa- ter flux update. An example of this oscillation is shown and discussed in the next section (Test 4a).

The key component in our flow routing scheme is calculating the probabilities for routing water parcels, based on rules abstracting the governing physics - hence the name weighted random walk. As the detailed procedure is explained in Part 1, here we only revisit the main idea. The likelihood of a neighbor cell receiving a water parcel is determined by a combination of local quantities including water surface gradient, the direction of flow inertia, and flow depth at the cell.

The steps for calculating the routing probability field are described below.

\subsection{Step 1: define a routing direction at each cell}

The routing direction $(\boldsymbol{F})$ is essentially an estimate of local downstream direction for the purpose of directing the water flux. In our model, it is a combination of the estimated local water surface gradient and direction of flow inertia:

$$
\begin{aligned}
& \boldsymbol{F}^{*}=\gamma \boldsymbol{F}_{\mathrm{sfc}}+(1-\gamma) \boldsymbol{F}_{\mathrm{int}}, \\
& \boldsymbol{F}=\frac{\boldsymbol{F}^{*}}{\left|\boldsymbol{F}^{*}\right|},
\end{aligned}
$$

where $\boldsymbol{F}_{\text {int }}=\boldsymbol{q}|\boldsymbol{q}|$ is a unit vector indicating inertia, and $\boldsymbol{F}_{\mathrm{sfc}}=\frac{\nabla H}{|\nabla H|}$ is a unit vector indicating surface gradient. Both $\boldsymbol{q}$ and $\nabla H$ take value from the latest iteration step. $\gamma$ is a dimensionless coefficient, which is set to 0.05 in our runs if not indicated otherwise (Liang et al., 2015).

The value of $\gamma$ affects the results of FlowRCM by controlling how sensitive the flow's response is to the water surface slope. For example, in the case of a distributary network, the higher concentration of water flux in channels will result in a slightly higher water surface profile than that of the surrounding floodplain, which causes a lateral surface gradient pointing away from the channels to the floodplains. This mechanism controls how much flow "escapes" from channels and spreads onto the surrounding floodplain. A guideline for the 
choice of the value of $\gamma$ for a range of environments, including steeper terrains such as alluvial fans, can be obtained by expressing $\gamma$ as a function of the characteristic slope and flow velocity of the environment, and grid size:

$\gamma_{0}=\frac{g \delta_{\mathrm{c}} S_{0}}{U_{0}^{2}}$,

where $g$ is the gravitational acceleration, $\delta_{\mathrm{c}}$ is the grid size, $S_{0}$ is the characteristic slope of the system, and $U_{0}$ is the characteristic flow velocity. The characteristic slope can be estimated by the average topographic or water surface slope of the system, and the characteristic flow velocity can be estimated as the mean velocity of the major channels. Equation (3) directly takes into account the steepness of the environment as well as the grid size. Using Wax Lake delta (WLD) - a subdelta of the Mississippi River delta system as an example, the characteristic slope is $5 \times 10^{-5}$, grid size is $60 \mathrm{~m}$ (see Test $4 \mathrm{a}$ ) and the characteristic flow velocity from field surveys is $0.5-1 \mathrm{~m} \mathrm{~s}^{-1}$ depending on the flooding stage, resulting in a range of $\gamma$ values from 0.03 to 0.12 . The choice of 0.05 is within this range. A quantitative analysis of the effects of $\gamma$ on the modeling results can be found in Test $4 a$.

\subsection{Step 2: calculate relative routing weights for the neighbors at each cell}

With the routing direction $\boldsymbol{F}$ specified, the relative routing weights $\left(w_{i}\right)$ for neighbors around each cell (eight neighbors in the case of square lattice setup) are determined as follows:

$w_{i}=\frac{h_{i} \max \left(0, \boldsymbol{F} \cdot \boldsymbol{d}_{i}\right)}{\Delta_{i}}, i=1, \ldots, 8$.

The cellular direction vector, $\boldsymbol{d}_{i}$, is a unit vector pointing to neighbor $i$ from the given cell, $\Delta_{i}$ is the cellular distance, taking a value of 1 for cells in main compass directions and a value of $\sqrt{2}$ for corner cells, and $h_{i}$ is water depth of neighbor $i$.

\subsection{Step 3: calculate routing probabilities $\left(p_{i}\right)$}

The weights above are then processed according to the wetdry status of cells. The model considers cells with flow depth greater than a threshold value, typically $1-5 \%$ of the characteristic flow depth of the system, as "wet" cells, and the opposite as "dry" cells. The weights for dry cells are then converted to the value of 0 . The routing probabilities $\left(p_{i}\right)$ are calculated as

$p_{i}=\frac{w_{i}}{\sum_{\mathrm{nb}=1}^{8} w_{\mathrm{nb}}}, i=1, \ldots, 8$,

where "nb" is the numbering of neighbors around a given cell (1-8 for a 3-by-3 square grid). With the routing probabilities calculated, water parcels are released one by one from the upstream inlet cells and follow a weighted random walk based on the probability field. The cumulative movements of parcels are summed in terms of vectors at each cell to obtain an estimation of flow unit discharge. The direction is given by the average walking direction of the passing parcels and the magnitude is given by the summation of the fluxes carried by these parcels.

The calculation of the water surface profile is performed with a 1-D scheme along the water parcel paths, rather than the solution of a system of partial differential equations. The basic assumption of this approach is that the water surface profile along a streamline can be approximated by a 1-D equation. In the morphodynamic results in Part 1, this 1-D equation takes the simplest form that the water surface slope on the delta equals a constant value - the "reference slope". For the hydrodynamic tests in this work, we formulate the 1-D equation such that (i) it satisfies the backwater equation if the local Froude number is low $\left(F r^{2} \leq 0.5\right)$ and (ii) the water surface slope $(S)$ is equal to the friction slope $\left(S_{\mathrm{f}}\right)$ if the Froude number is high $\left(\mathrm{Fr}^{2}>0.5\right)$ :

$$
\begin{aligned}
& \frac{\partial h}{\partial l}=\frac{S-S_{\mathrm{f}}}{1-F r^{2}}, \text { if } F r^{2} \leq 0.5, \\
& \frac{\partial H}{\partial l}=S_{\mathrm{f}}, \text { if } F r^{2}>0.5,
\end{aligned}
$$

where $C_{\mathrm{f}}$ is the coefficient of friction, $S_{\mathrm{f}}=C_{\mathrm{f}} F r^{2}=C_{\mathrm{f}} \frac{U^{2}}{g h}$ is the friction slope and $l$ is the distance along an arbitrary flow streamline.

This calculation is done along each water parcel path via a finite difference scheme. An average value is taken for cells belonging to multiple paths. The obtained surface profile is then smoothed with numerical diffusion to remove bumps and ditches caused by the gaps between different 1-D paths. In addition, an underrelaxation is applied for numerical stability between iterations (see Part 1 for details). This method assumes that along the streamline the flow is 1-D so that Eqs. (6) and (7) apply. However, the combination of a large number of flow streamlines covering many flow paths essentially constitutes a 2-D surface. This surface calculation does not work for strongly varying 2-D flows, but in the tests below we will show that this highly simplified surface calculation is sufficient to reproduce large-scale flow partitioning, a very important application for deltaic distributary networks.

Using this parcel-based flow routing method, flow continuity is always satisfied because (1) there is no gain or loss of the water flux represented by each water parcel, and (2) at each cell in the domain the number of parcels coming in is always equal to the number of parcels leaving that cell. Equations (6) and (7) not only use the streamlines identified from the new unit discharge field, but also use the value of discharge itself to update the water surface profile. During this process, the model assumes that the discharge at each cell remains the same, while the depth and velocity are updated according to the new water surface elevation. Therefore flow continuity remains satisfied. 
FlowRCM is written in Matlab and typically runs in $1-2 \mathrm{~h}$ on a personal laptop for simulations in the tests presented in the next section. The running speed could be reduced by an order of magnitude if the code were written in more efficient languages (such as $\mathrm{C}$ ) and optimized.

\section{Hydrodynamic test cases}

The hydrodynamic test cases are designed to analyze two important aspects in the RCM. First, we target the "reducedcomplexity" features in our flow routing model, i.e., the rules and parameterizations that represent governing physics; second, we want to capture critical hydrodynamic processes affecting the overall delta morphology. The hydrodynamics of river deltas involve a hierarchy of processes occurring at a wide range of scales, from flow structures behind individual ripples on the bed to channel avulsions that change the distribution of water flux across the entire delta surface. We focus on hydrodynamic processes at the channel scale, which are essential to creating, maintaining and modifying a distributary channel network. The proposed tests are a (1) backwater profile in a straight channel, (2) flow around a mouth bar, (3) partitioning of flow at a single bifurcation, and (4) partitioning of flow in a distributary channel network with submerged islands. FlowRCM results are compared to Delft3D outputs and theoretical predictions. The description and results of each test case are given below. All Delft3D simulations were done in depth-averaged mode, with a time step of $30 \mathrm{~s}$ and a constant horizontal eddy viscosity $\left(1 \mathrm{~m}^{2} \mathrm{~s}^{-1}\right)$.

\subsection{Test 1: backwater profile in a straight channel}

River deltas have subtle topography and water surface gradients and flow is typically subcritical. Nevertheless, the gradient in the surface profile is essential to water motion in deltaic environments (Edmonds and Slingerland, 2008). As mentioned in the previous section, FlowRCM estimates the water surface based on the assumption of a 1-D surface profile along flow streamlines. As the movement of water parcels on the grid is in both $x$ and $y$ directions, usually in a "zigzag" fashion and not necessarily along flow streamlines, the calculation of water surface along the flow paths involves projecting each step a parcel makes to the estimated streamline. Thus, in the first test we use a straight channel of constant width with no variation in cross-stream direction, whose surface can be described by the 1-D backwater equation. The model performance is compared to the theoretical backwater profile.

The domain is $2000 \mathrm{~m}$ wide and $15000 \mathrm{~m}$ long with a rectangular cross section (Fig. 2a). The bed has a constant slope $S=10^{-3}$ and a constant friction coefficient $C_{\mathrm{f}}=0.01$. A constant discharge of $2 \times 10^{4} \mathrm{~m}^{3} \mathrm{~s}^{-1}$ is fed into the upstream inlet. The downstream outlet has a fixed water surface elevation as boundary condition. The discharge is chosen so that the flow remains subcritical in normal flow conditions.

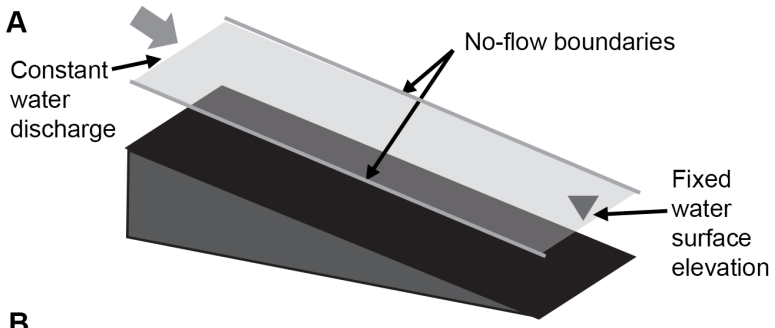

B

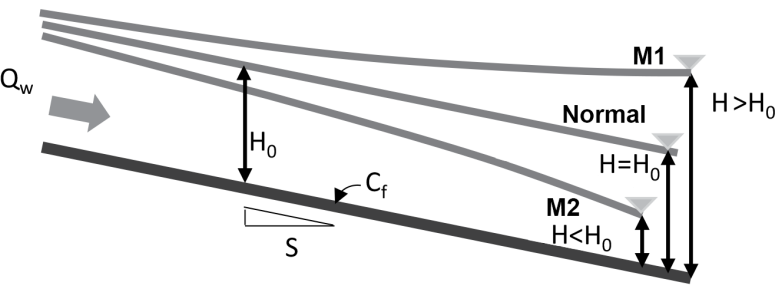

Figure 2. The setup of test 1 (straight channel test). (a) The channel has a rectangular cross section, constant bed slope and friction coefficient. A constant discharge is fed into the upstream inlet. A fixed water surface elevation boundary condition is assigned at the downstream outlet. (b) Three backwater profiles (M1, normal, and M2) are shown in the bottom diagram where downstream water surface elevation is respectively above, equal to, or below that of normal flow.

By varying the downstream water surface elevation relative to that of the normal flow, different backwater profiles can be achieved, such as M1, M2 and normal flow (Chow, 1959) (Fig. 2b). As the flow does not have cross-stream variation, the surface profile can be resolved using the 1-D backwater equation (Eq. 6).

For the FlowRCM calculation, we use a grid of $20 \times 150$ cells, with cell size of $100 \mathrm{~m}$. The water surface elevation calculated from FlowRCM is averaged in the crossstream direction and compared to the numerical solution of Eq. (6).

The test results show that in all three scenarios FlowRCM is able to successfully reproduce the backwater profile solution (Fig. 3). The predicted longitudinal water surface profile (averaged in the cross-stream direction), in fact, matches the solution of the 1-D backwater equation. The simple scheme used in FlowRCM is thus able to produce realistic water surface profiles.

\subsection{Test 2: flow around a mouth bar}

The formation of channel bifurcations is essential to the formation of the distributary channel network on river deltas (Edmonds and Slingerland, 2007; Kleinhans et al., 2013). Therefore, the ability of a numerical model to represent the bifurcation process is of great importance. As shown by the work of Edmonds and Slingerland (2007), mouth-bar development is critical to channel bifurcation. Particularly, one key feature in this process is the transition from flow acceleration to deceleration over the top of the mouth bar as 

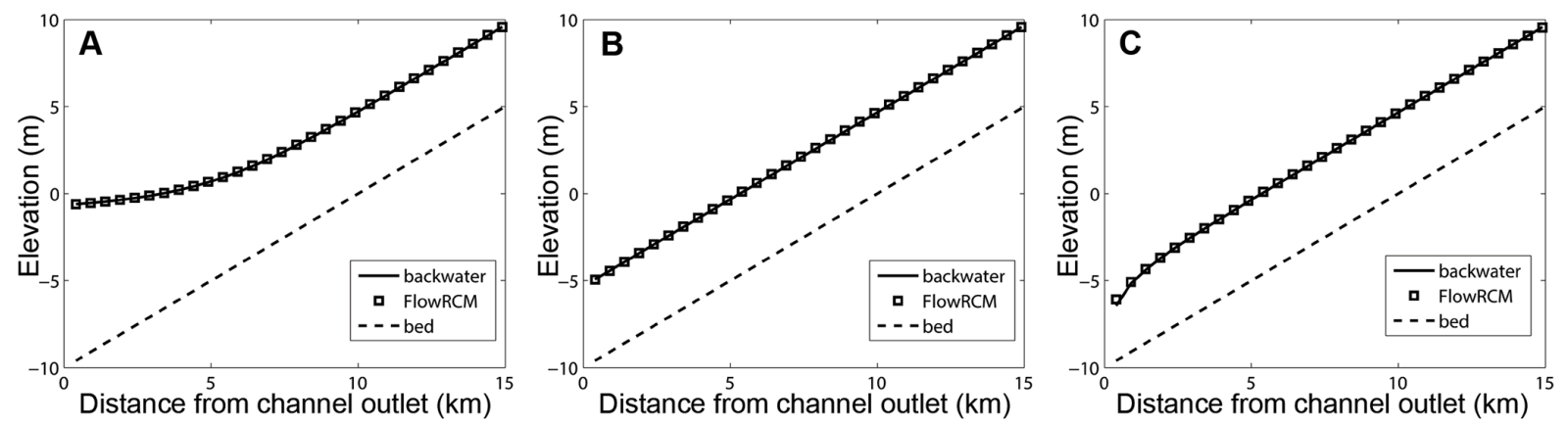

Figure 3. Results from test 1 (straight channel test). The water surface profile in the longitudinal direction calculated by FlowRCM (averaging the 2-D profile in the cross-stream direction) is compared to the numerical solution of the 1-D backwater equation in three scenarios: (a) M1 curve, (b) normal flow, and (c) M2 curve.

the bar height grows and the bar top approaches the water surface (Fig. 4). Here we design a similar test with variable bar heights. The mouth-bar topography is represented by a Gaussian-shaped bump in a straight channel. The bump does not deform and its height is varied from 10 to $99 \%$ of the normal flow depth.

The domain is a straight channel section, $100 \mathrm{~m}$ wide and $200 \mathrm{~m}$ long with rectangular cross section. The bed has a constant slope $S=2.5 \times 10^{-3}$ and a constant friction coefficient $C_{\mathrm{f}}=0.1$. A constant water discharge of $50 \mathrm{~m}^{3} \mathrm{~s}^{-1}$ is fed into the upstream inlet. The downstream outlet has a fixed water surface elevation as downstream boundary condition. The Gaussian-shaped bump has a diameter equal to approximately one third of the channel width. The side walls of the channel have a no-flow boundary condition. Both FlowRCM and Delft3D use a $40 \times 80$ grid with cell size of $25 \mathrm{~m}$.

We compare the outputs from FlowRCM and Delft3D in terms of water surface elevation and flow velocity, focusing on (i) the location of "hot spots" of high and low velocity (ii) the deformation of the water surface in proximity of the bump, and (iii) the transition of flow velocity right over the top of the bump as the bump height increases from 10 to $99 \%$ of the normal flow depth.

The maximum flow velocity over the top of the bump occurs for a bump height of around $60 \%$ of the flow depth (Edmonds and Slingerland, 2007). Therefore, we compare the outputs of FlowRCM and Delft3D for bump heights equal to 20,60 , and $90 \%$ of the flow depth (Fig. 5). The results show that (i) both FlowRCM and Delft3D reproduce the transition from flow acceleration to deceleration over the top of the bump as the height of the bump increases (Figs. 5, 6); (ii) both models show that higher velocity occurs in a "bow shape" over the bump and curved into the downstream direction, while lower velocity occurs behind the bump and slightly in front of the bump (Fig. 5); and (iii) both models show that the water surface is superelevated in front of the bump and is drawn down behind the bump. FlowRCM thus captures the key hydrodynamic features, although their magnitude and shape do not exactly match the results from
A
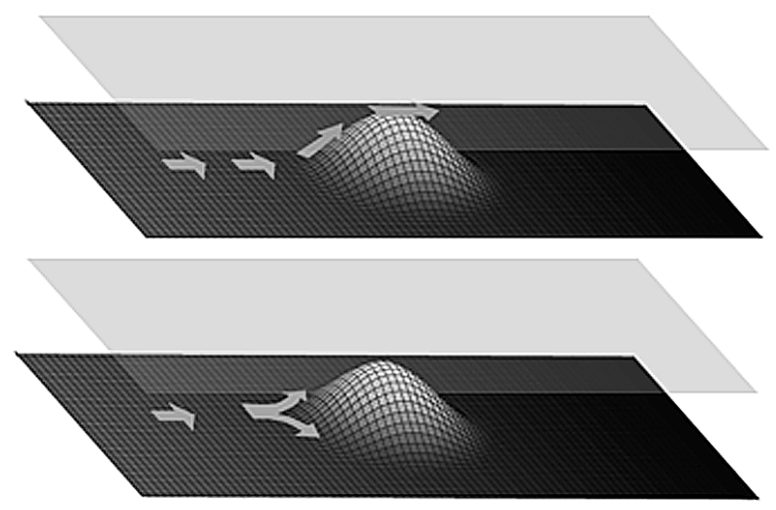

B

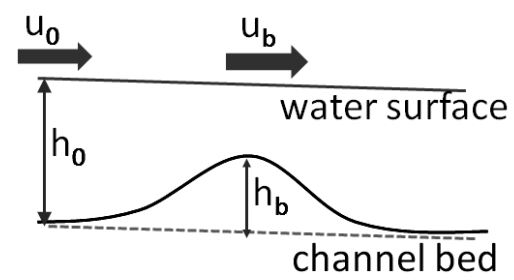

Figure 4. Setup of test 2 (flow around a mouth bar). A smooth Gaussian-shape bump is placed in a straight channel. (a) Two scenarios are observed: (i) acceleration over the bump or, (ii) deceleration over the bump and diversion around the bump. (b) Sketch of the key variables: normal flow depth $\left(h_{0}\right)$ and velocity $\left(u_{0}\right)$, bump top velocity $\left(u_{\mathrm{b}}\right)$, and bump height $\left(h_{\mathrm{b}}\right)$.

Delft3D (Fig. 5). The irregularities in the FlowRCM output are caused by the "randomness" inherent in the weighted random walk scheme. As seen in Figs. 4 and 5 such irregularities do not affect the ability of the model to reproduce key hydrodynamic features.

\subsection{Test 3: flow through a single bifurcation}

Bifurcations control the partitioning of water and sediment in the deltaic system and affect the stability of the whole 


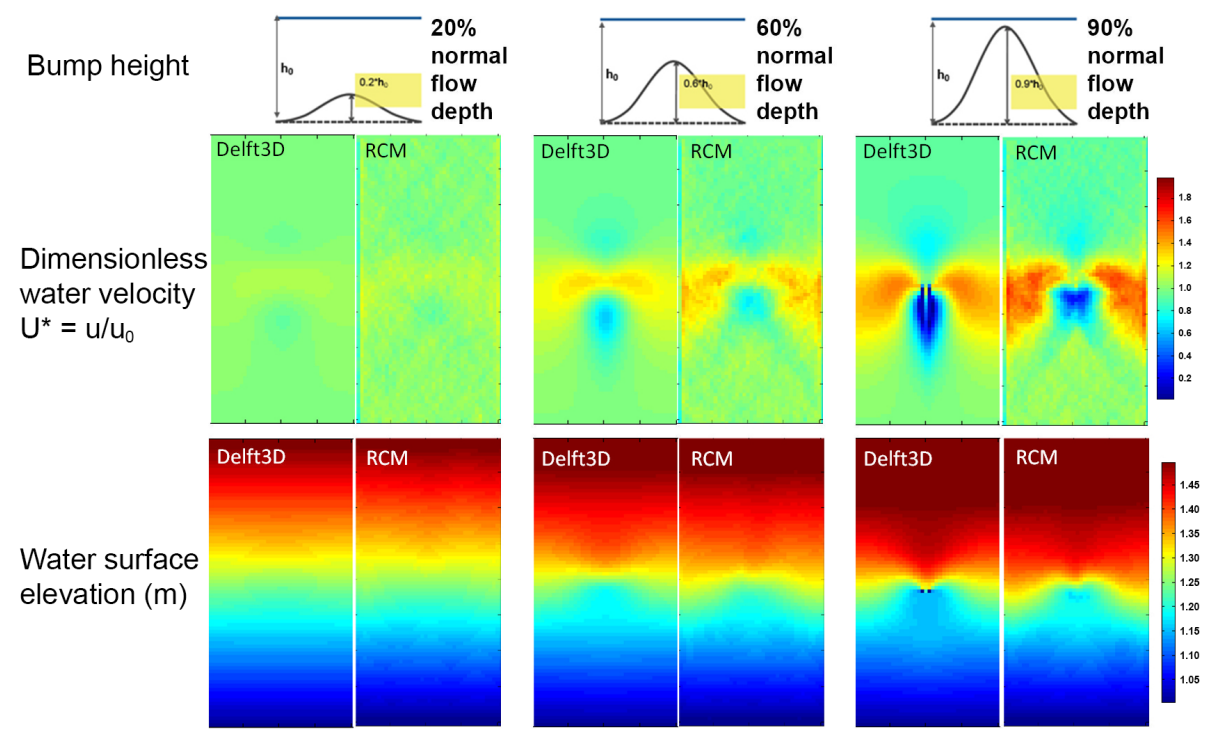

Figure 5. Contour plots of water velocity magnitude (scaled by the normal flow velocity $u_{0}=0.5 \mathrm{~m} \mathrm{~s}^{-1}$ ) and water surface elevation for three cases (bump heights at 20,60 and $90 \%$ of normal flow depth). Flow direction is from top to bottom. Notice the deformation of the water surface and the development of a low velocity region in front of and behind the bump.

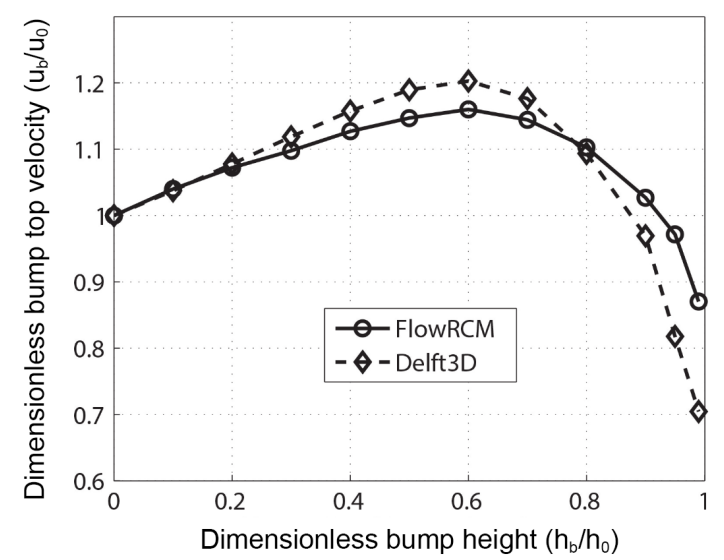

Figure 6. Comparison of dimensionless bump top velocity $\left(u_{\mathrm{b}} / u_{0}\right)$ as a function of dimensionless bump height $\left(h_{\mathrm{b}} / h_{0}\right)$ predicted by FlowRCM and Delft3D. Both models predict an initially rising and then falling flow velocity as the dimensionless bump height goes to 1 . FlowRCM also captures the turning point where rising changes to falling ( $\sim 60 \%$ flow depth). Flow velocity is scaled by the normal flow velocity magnitude, and bump height by normal flow depth.

network (Edmonds and Slingerland, 2008; Edmonds et al., 2010; Kleinhans et al., 2008, 2013). We use a simple and idealized bifurcation topography to test the response of FlowRCM to bifurcation asymmetry, e.g., changes in width and/or depth ratio of the two downstream branches.

There are three groups of tests, referred to as group A, B and $\mathrm{C}$. In all groups the main channel splits into two branches that open at an angle of $60^{\circ}$, and both branches enter a basin with constant depth (Fig. 7). Channel banks are no-flow

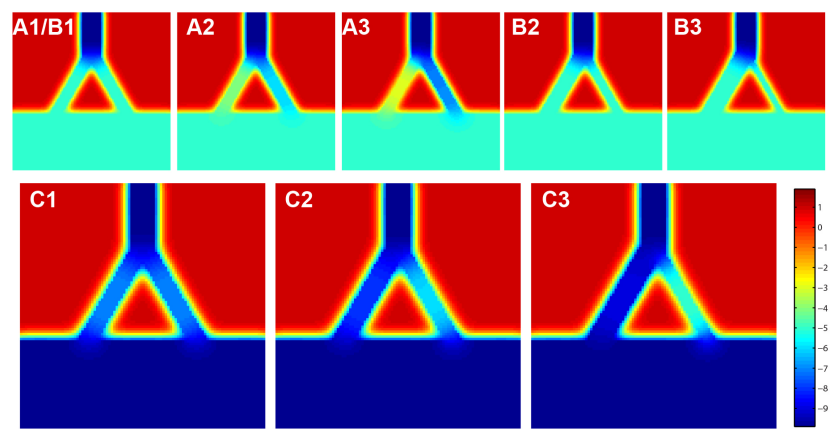

Figure 7. Channel geometries for test 3 (single bifurcation test). Group A (A1, A2 and A3) and group B (B1, B2 and B3) have a smaller domain and input discharge. Within group $A$ the ratio of depth between the two downstream branches is varied while keeping width constant. Within group B the ratio of width between the two downstream branches is varied while keeping depth constant. Within group $\mathrm{C}(\mathrm{C} 1, \mathrm{C} 2$ and $\mathrm{C} 3)$ width and depth are varied in the same proportion while keeping the summation of cross-sectional area constant.

boundaries so that the water flux stays in the channel and there is no flooding onto the bank. For all tests, FlowRCM and Delft3D use a 100 by 100 grid. The tests in groups $\mathrm{A}$ and B have smaller domain sizes of $2500 \mathrm{~m}$ by $2500 \mathrm{~m}$ with $25 \mathrm{~m}$ grid size, and total input discharge of $3000 \mathrm{~m}^{3} \mathrm{~s}^{-1}$. The tests in group C have larger domain sizes of $5000 \mathrm{~m}$ by $5000 \mathrm{~m}$ with $50 \mathrm{~m}$ grid size, and total input discharge of $5000 \mathrm{~m}^{3} \mathrm{~s}^{-1}$. In group A we explore the effect of asymmetry in depth alone, keeping the two branches at the same width; in group B the effect of asymmetry in width alone, keeping the two branches at same depth; and in group $\mathrm{C}$ the effect of 
Table 1. Discharge asymmetry and upstream surface elevation results from FlowRCM and Delft3D for all single bifurcation runs.

\begin{tabular}{crrrrrrrr}
\hline \multirow{2}{*}{ Run } & \multicolumn{3}{c}{ Discharge asymmetry $(\%)$} & & \multicolumn{3}{c}{ Upstream surface elevation $(\mathrm{m})$} \\
\cline { 2 - 3 } \cline { 7 - 8 } & FlowRCM & Delft3-D & Difference & & FlowRCM & Delft3-D & Difference \\
\hline A1 & $0.16 \%$ & $0.00 \%$ & $+0.16 \%$ & & 0.3226 & 0.2067 & +0.1159 \\
A2 & $20.82 \%$ & $24.04 \%$ & $-3.23 \%$ & & 0.3160 & 0.2048 & +0.1112 \\
A3 & $41.04 \%$ & $47.69 \%$ & $-6.65 \%$ & & 0.3156 & 0.2016 & +0.1140 \\
B1 & $0.16 \%$ & $0.00 \%$ & $+0.16 \%$ & & 0.3226 & 0.2067 & +0.1159 \\
B2 & $21.59 \%$ & $21.17 \%$ & $+0.42 \%$ & & 0.3230 & 0.2068 & +0.1162 \\
B3 & $40.76 \%$ & $42.78 \%$ & $-2.02 \%$ & & 0.3301 & 0.2075 & +0.1226 \\
C1 & $0.21 \%$ & $0.01 \%$ & $+0.19 \%$ & & 0.1472 & 0.1091 & +0.0381 \\
C2 & $28.22 \%$ & $27.84 \%$ & $+0.37 \%$ & & 0.1417 & 0.1057 & +0.0360 \\
C3 & $51.49 \%$ & $52.66 \%$ & $-1.17 \%$ & & 0.1286 & 0.0963 & +0.0323 \\
\hline
\end{tabular}

asymmetry in cross-sectional area, by keeping the summation of the cross-sectional areas constant and all channels at the same width-to-depth ratio. Detailed channel geometries are shown in Fig. 7.

The output of FlowRCM is compared to that of Delft3D in terms of (i) the spatial pattern of flow velocity and water surface elevation and (ii) the ratio of fluxes between the two branches.

To evaluate the effect of channel geometry asymmetry on flow partitioning between bifurcations, we calculate two values from each test result for both FlowRCM and Delft3D, namely the asymmetry of discharge $\psi_{Q}$ and the asymmetry of cross-sectional area $\psi_{\mathrm{A}}$ :

$\psi_{Q}=\frac{\left|Q_{\mathrm{L}}-Q_{\mathrm{R}}\right|}{Q_{\mathrm{L}}+Q_{\mathrm{R}}}$,

$\psi_{\mathrm{A}}=\frac{\left|A_{\mathrm{L}}-A_{\mathrm{R}}\right|}{A_{\mathrm{L}}+A_{\mathrm{R}}}$,

where $Q_{\mathrm{L}}$ and $Q_{\mathrm{R}}$ are the water discharge in the left and right branches respectively; $A_{\mathrm{L}}$ and $A_{\mathrm{R}}$ are the cross-sectional area (calculated with bed elevation and resolved water surface elevation) of the left and right branches respectively.

The asymmetry values defined in Eqs. (8) and (9) are plotted in Fig. 8. FlowRCM captures the same trend of flow discharge partitioning predicted by Delft3D: the amount of water flux into the two branches is proportional to the crosssectional area of the branches. This means that despite the variation in the ratio of depth and/or width, the flow tends to have the same mean velocity in both branches. Also, both FlowRCM and Delft3D predict a water surface gradient in the shallower branch that is significantly higher than the one in the deeper branch, consistent with field observations by Edmonds and Slingerland (2008). An example of flow field and water surface elevation (test C3) is plotted in Fig. 9. Also, within each test group both FlowRCM and Delft3D predict the same behavior of upstream water surface elevation in response to the discharge asymmetry: in groups $\mathrm{A}$ and $\mathrm{C}$ the upstream water surface elevation decreases as discharge

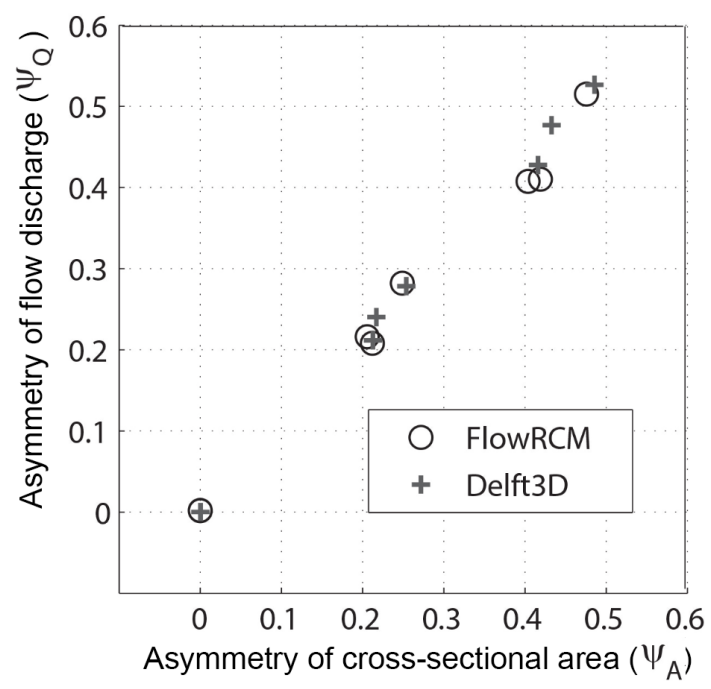

Figure 8. Comparison of discharge asymmetry as a function of cross-sectional area asymmetry predicted by FlowRCM and Delft3D. Both models result in asymmetry of discharge proportional to the asymmetry of cross-sectional area.

asymmetry increases; in group B the upstream water surface elevation increases as discharge asymmetry increases.

Qualitative differences in the observed flow plan-view pattern produced by FlowRCM and Delft3D emerge due to what we call "local effects". In the results of FlowRCM there is significant concentration of flux right upstream of the island, where flow bifurcates directly against the tip of the island (in this case, the no-flow condition at the boundary of the islands does not allow any water flux to escape the channels) (Fig. 9, dashed square region). The water surface elevation calculated by FlowRCM does not show superelevation right at the tip of the island as in the Delft3D results; instead, there is a drawdown. We believe that the superelevation creates a gradient that causes the flow to divert around the island much further upstream. 
A

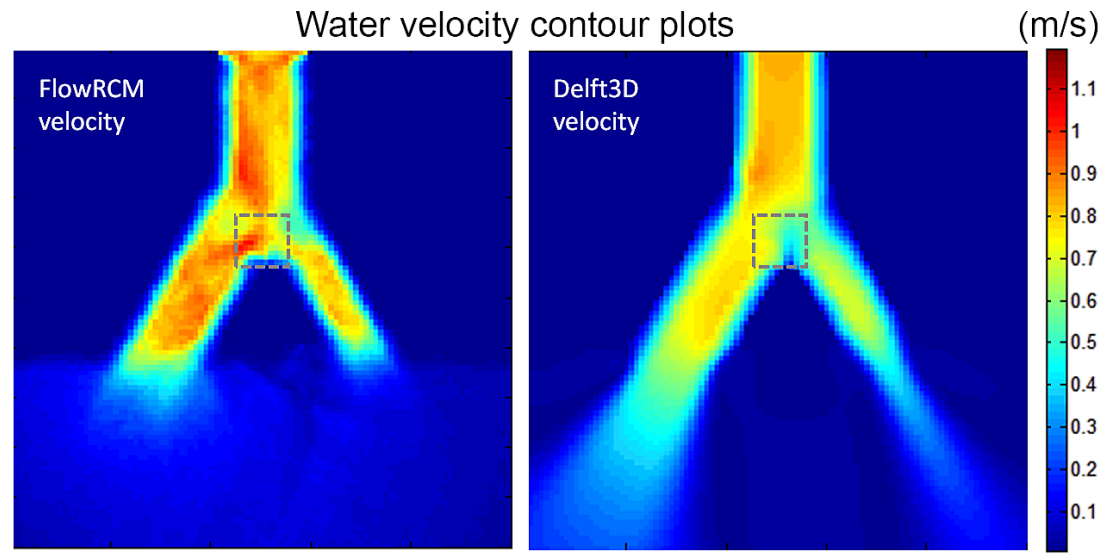

B

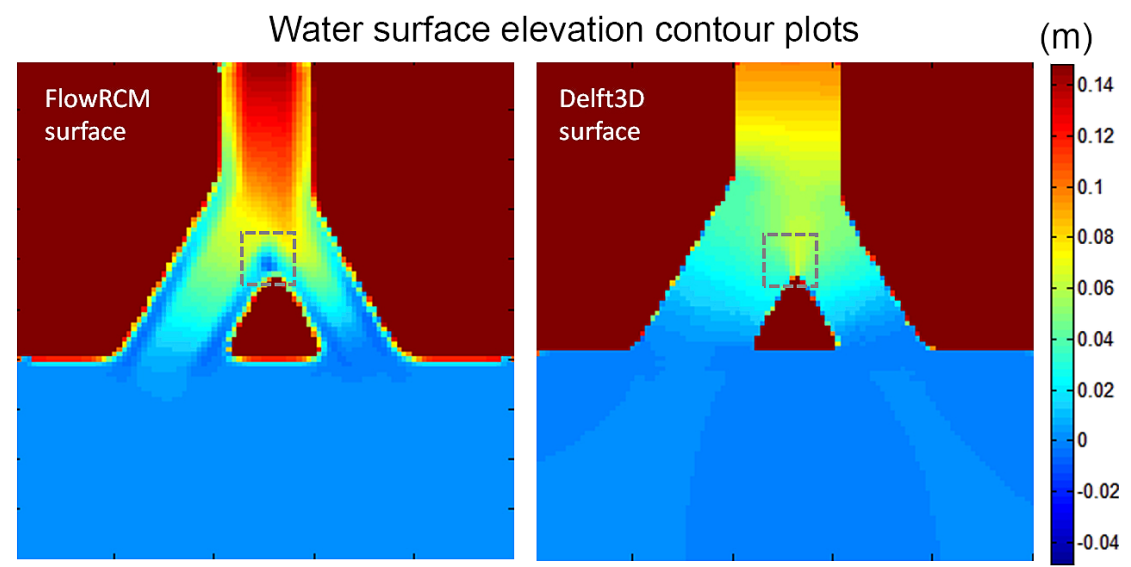

Figure 9. Results from run C3: (a) water velocity and (b) water surface elevation. FlowRCM and Delft3D behave differently at the upstream side of the triangle island (marked by the dashed gray square): while Delft3D exhibits a low velocity zone with a high water surface elevation, FlowRCM exhibits a high velocity zone and a low water surface elevation.

In quantitative terms, this single-bifurcation test shows that FlowRCM predicts a discharge asymmetry within $7 \%$ of difference compared to Delft3D, but tends to overpredict surface elevation by up to $50 \%$ (Table 1). As discussed in the companion paper, the inclusion of water surface is required but its accuracy can be relaxed when modeling deltas. The error in the calculation of the surface elevation is due to the fact that FlowRCM calculates the discharge vector at each cell by (1) summing up the volume of all passing water parcels at each time step to obtain the magnitude of the discharge vector, and (2) doing a vector summation of the moving directions of all passing water parcels (each will be a unit direction pointing to one of the eight neighboring cells) to obtain the direction of the discharge vector. By doing so, in the area where there is flux convergence, some cross-stream flux caused by the parcels traveling almost sideways to the main flow direction will be counted as contributing to the total downstream flux as well, causing an overestimation of the water surface elevation. An alternative method would be directly doing a vector summation of the discharge vector represented by each passing parcel; however, in this way, discharge and water surface elevation would be underpredicted.
Despite this effect, the parcel-based Lagrangian approach is able to predict flow partitioning at a bifurcation and offers more flexibility in flow routing in complex terrains with a low wet-to-dry ratio and frequent changes in wetted area.

\subsection{Test 4: flow through a distributary channel network}

We now evaluate the performance of FlowRCM at the scale of a complete deltaic distributary channel network. We are also interested in assessing whether the "inaccuracy" of the flow field at finer scales accumulates as the flow propagates through the whole channel network. The topography setups used to run FlowRCM and Delft3D are the synthetic topography of a natural river delta (Test 4a) and a DeltaRCMsimulated topography (Test $4 \mathrm{~b}$ ). By introducing a whole distributary network, which has no constrains on lateral flux exchange between channel and islands, we essentially test the behavior of FlowRCM in a transition from confined to unconfined flow. Both tests $4 \mathrm{a}$ and $4 \mathrm{~b}$ use a distributary network that features flow spreading from a single feeding channel which is strictly confined by nonpenetration high walls to an approximately $90^{\circ}$ or $180^{\circ}$ open space. Water not only flows 
Table 2. Effect of the parameter $\gamma$ on the flux distribution between channels and islands. Detailed cross-section locations are given in Fig. 14.

\begin{tabular}{lc}
\hline$\gamma$ & $Q_{\text {channel }}^{1} / Q_{\text {island }}^{2}$ \\
\hline 0.025 & 1.2554 \\
0.05 & 1.1417 \\
0.075 & 1.0161 \\
0.1 & 0.9713 \\
\hline${ }^{1} Q_{\text {channel }}=Q_{2}+Q_{4}+Q_{6}+Q_{8}$ \\
${ }^{2} Q_{\text {island }}=Q_{1}+Q_{3}+Q_{5}+Q_{7}+Q_{9}$
\end{tabular}

through the channel network but also onto the islands. Therefore the whole delta surface, except for the upstream tips of a few islands closer to the apex, is inundated.

\subsubsection{Test 4a: synthetic Wax Lake delta (WLD) topography}

The WLD is a modern river-dominated delta in the coast of Louisiana, part of the Mississippi River delta system (Roberts et al., 1980; Wellner et al., 2005; Shaw et al., 2013). We construct a synthetic bathymetry (Fig. 10) from satellite images and bathymetry measurements along nine transects (USACE, 1999). We use an image processing software (Adobe Photoshop CS6) to integrate the plan view of islands and channels from the satellite imagery and obtain a smooth profile between neighboring transects. FlowRCM and Delft3D are given the same initial and boundary conditions: an upstream inlet channel discharge of $2490 \mathrm{~m}^{3} \mathrm{~s}^{-1}$ and a water surface elevation at the downstream boundary of $0 \mathrm{~m}$ mean sea level. The friction coefficient is set to 0.01 . Both FlowRCM and Delft3D simulations for this test case use a 200 by 200 grid with a cell size of $60 \mathrm{~m}$.

The results show that the two models predict similar flow distribution among channels and islands with "hot spots" of higher flow velocity occurring at the same locations (Fig. 11). Delft3D shows a more "diffused" velocity map while FlowRCM gives a more "noisy" map. The flow pattern on the islands predicted by FlowRCM seems to respond to island topography, exhibiting a converging stream in the low elevation area towards the lower center of the islands (Fig. 11). In Delft3D results, flow is more evenly distributed across each island, thus not revealing much of the island's topographic detail (although higher-resolution Delft3D modeling would, while constraints exist in its drying-flooding algorithm). The depth-averaged mode and the choice of horizontal eddy viscosity might also add to the relatively smooth outputs from Delft3D. The "local effects" in FlowRCM discussed in the previous section cause the appearance of a high velocity zone right along the tip of the islands rather than a low velocity zone caused by flow diversion starting upstream of the island as in the Delft3D results (Fig. 12).

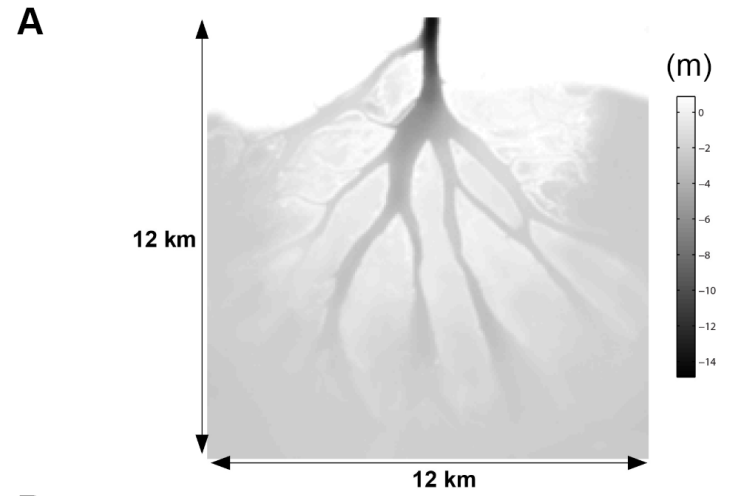

B

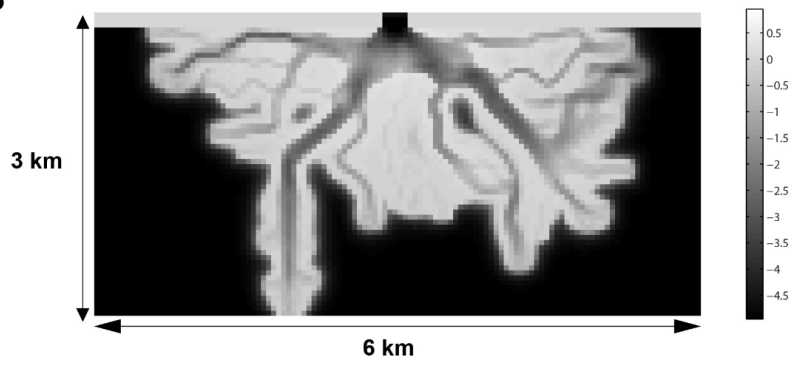

Figure 10. Topography used in tests $4 \mathrm{a}$ and $4 \mathrm{~b}$ (flow through a distributary channel network). (a) Synthetic topography constructed for WLD; (b) a DeltaRCM-generated delta topography.

Our reduced-complexity water surface calculation method requires a large underrelaxation to achieve smooth transition in the surface profile. This underrelaxation causes a delay between the response of the water discharge distribution to changes in water surface slope and, therefore, an oscillation in the cross-stream direction discharge distribution over 1020 iterations. By averaging over several iterations (e.g., the last 100 of a total 500 iterations) stable results are achieved, resulting in what we call a dynamic equilibrium. An example of this oscillation on the synthetic WLD topography is shown in Fig. 13.

To quantitatively evaluate the effect of the parameter $\gamma$, the setup of test $4 \mathrm{a}$ is used with values of $\gamma$ from 0.025 to 0.1 while keeping all other parameters unchanged. We measure the ratio of the discharge coming out of four main channels and the discharge coming out of the downstream side of the islands (Fig. 14). From the results listed in Table 2, it can be seen that with a higher value of $\gamma$, more flux is routed through the islands. In order to compare the flux distribution obtained with FlowRCM (for a value of the parameter $\gamma$ equal to 0.05 ) to results from Delft3D, we selected two transects located at different distances from the apex of the delta (Fig. 15). The closer transect, A, is divided into 10 sections and the farther transect, $\mathrm{B}$, is divided into 14 sections. The fraction of discharge at each section is calculated by the discharge crossing that section normalized by the total discharge crossing the whole transect. Results from FlowRCM and Delft3D are plotted for each transect. There is a close match between 

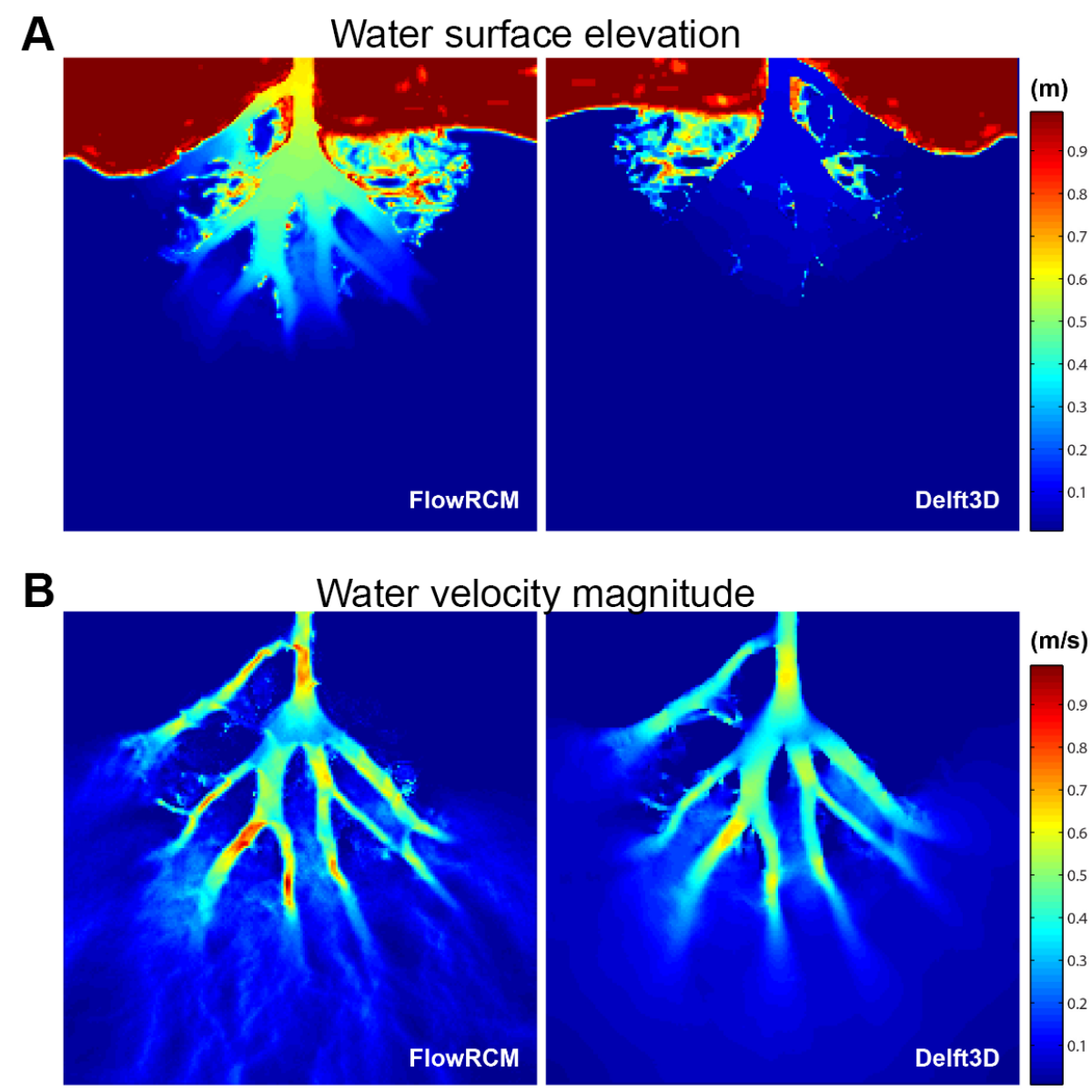

Figure 11. Results from test 4a (flow over synthetic bathymetry of WLD). (a) FlowRCM predicts a significantly higher gradient, while the water surface distribution is similar to the one predicted by Delft3D. (b) Velocity contour maps from model results; FlowRCM and Delft3D give similar flow distributions and predict "hot zones" where water velocity is significantly higher in channels.

A

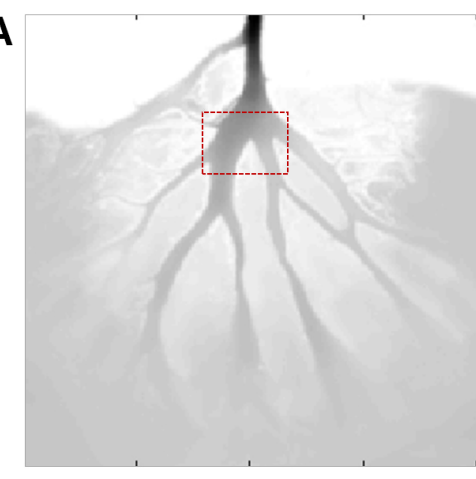

B

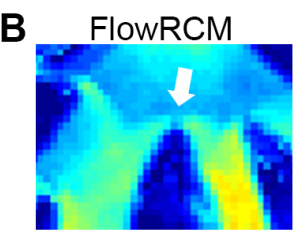

C

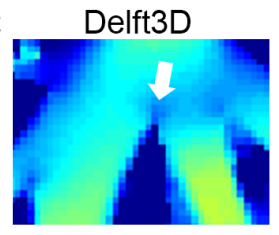

Figure 12. (a) The red dashed square marks one of the regions where FlowRCM displays strong local effects; (b) detailed view from both model results showing the "local effects" in FlowRCM, not present in the Delft3D simulation.

FlowRCM and Delft3D throughout transect A, while the results differ when flow reaches transect $\mathrm{B}$. The difference between transects A and B is due to the lateral flux normal to the main channel direction. FlowRCM has significantly less flux coming out of the very eastern and western sides of the delta $(<5 \%)$, compared to Delft3D $(>10 \%)$, resulting in a more concentrated flux distribution among the main channels and islands. Field surveyed data are needed to understand which model reproduces more closely the behavior of WLD.

\subsubsection{Test 4b: bed topography generated by DeltaRCM}

For this test, the delta topography is a snapshot of the mixed grain size delta simulated by DeltaRCM. The delta is composed of $70 \%$ fine grain and $30 \%$ coarse grain, and has a very well-defined distributary channel network (Liang et al., 2015) (Fig. 10). The rectangular domain is $6 \mathrm{~km}$ wide and $3 \mathrm{~km}$ long. The inlet channel has a discharge of $1250 \mathrm{~m}^{3} \mathrm{~s}^{-1}$, and the delta is formed in a basin with a constant depth of $5 \mathrm{~m}$. Both FlowRCM and Delft3D use a 60 by 120 grid with cell size of $50 \mathrm{~m}$. 
A

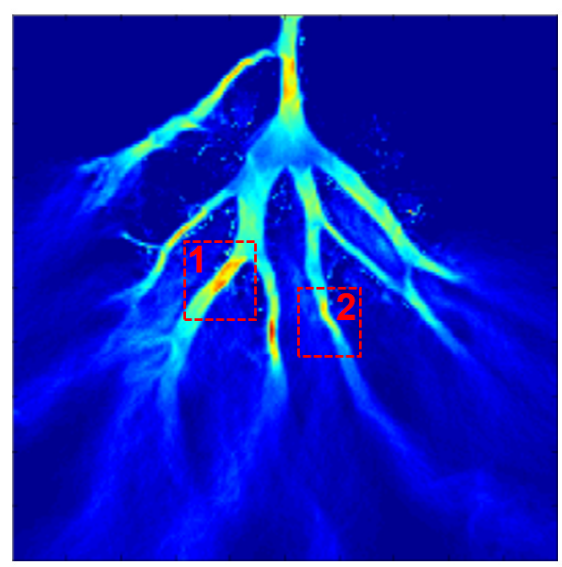

B

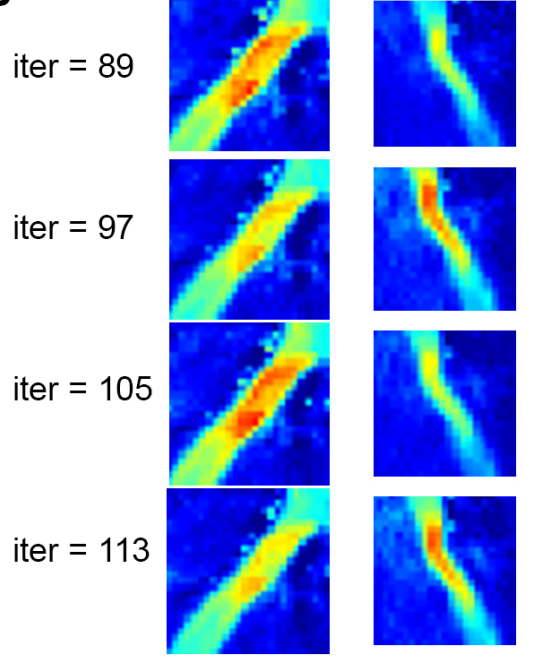

Figure 13. The dynamic balance between water surface gradient and flux distribution. (a) The dashed squares show focus areas enlarged on the right. (b) Oscillations of flow velocity between iterations in the focus areas.

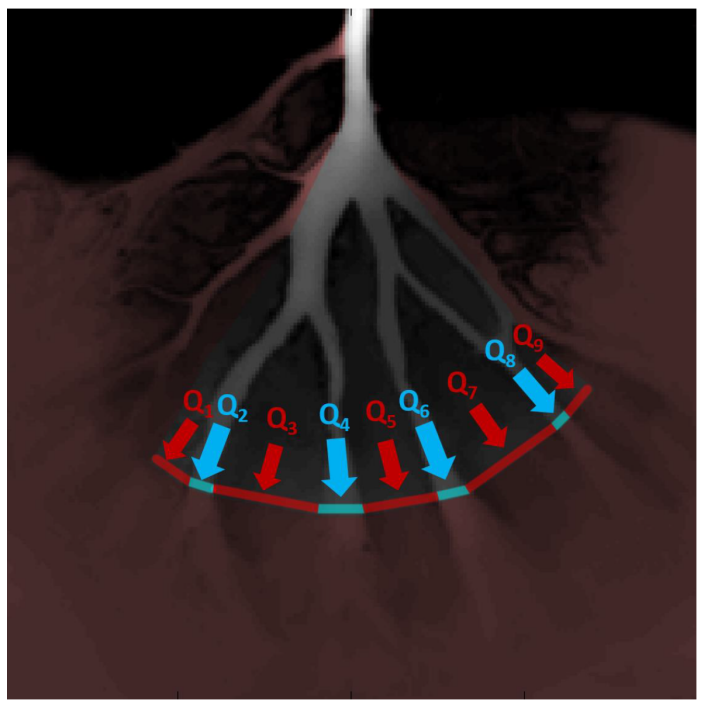

Figure 14. Setup used for analyzing the effect of the parameter $\gamma$ on modeling results. The ratio of the discharge coming out of four main channels $\left(Q_{2}, Q_{4}, Q_{6}, Q_{8}\right)$ and the discharge coming out of the downstream side of the islands $\left(Q_{1}, Q_{3}, Q_{5}, Q_{7}, Q_{9}\right)$ is measured to quantify how much flow is routed through the island as a function of the parameter $\gamma$.

These results show that, analogously to the synthetic WLD topography test, FlowRCM and Delft3D predict a similar flow distribution through the network, e.g., the partitioning among channels and islands (Fig. 16). Moreover, the water surface profile calculated by FlowRCM is smoother than the one obtained from Delft3D, as FlowRCM is able to handle discontinuities in the domain represented by frequent alternations of "wet" and "dry" cells. In this test "local effects" are not pronounced. A possible reason is that this simulated topography has already adapted to the features of the flow field calculated by FlowRCM, such that at the tip of the island local topography creates a smooth transition from deep channels to shallow islands that diverts the flow gradually. At the same time, since islands are mostly submerged and can be inundated, the calculation of the free surface allows for a more accurate surface construction compared to using a no-flow boundary condition at the edge of the islands.

\section{Discussion}

In this work, we evaluated the performance of FlowRCM in terms of its ability to reproduce plausible hydrodynamics of deltaic systems. We showed the performance of FlowRCM in several setups that mimic hydrodynamic scenarios characteristic of river deltas. The computed flow fields were compared to theoretical predictions and to numerical simulation results obtained with a higher-fidelity hydrodynamic model (Delft3D), previously validated for similar purposes (Lesser et al., 2004). From the comparison to analytical solutions and Delft3D, we address the goal stated in our introduction: what level of hydrodynamic physics does our FlowRCM capture? In terms of water surface profile modeling, FlowRCM is able to replicate (i) the backwater profile along a simple straight channel matching the solution of the theoretical backwater equation; (ii) the local surface deformation around submerged bumps, including a superelevated region upstream of the bump and a drawdown region downstream of the bump; (iii) the difference in surface gradients between two competing bifurcation branches; and (iv) the outward surface gradient from channels to floodplains. In terms of flow discharge and velocity field, FlowRCM is able to repli- 
A
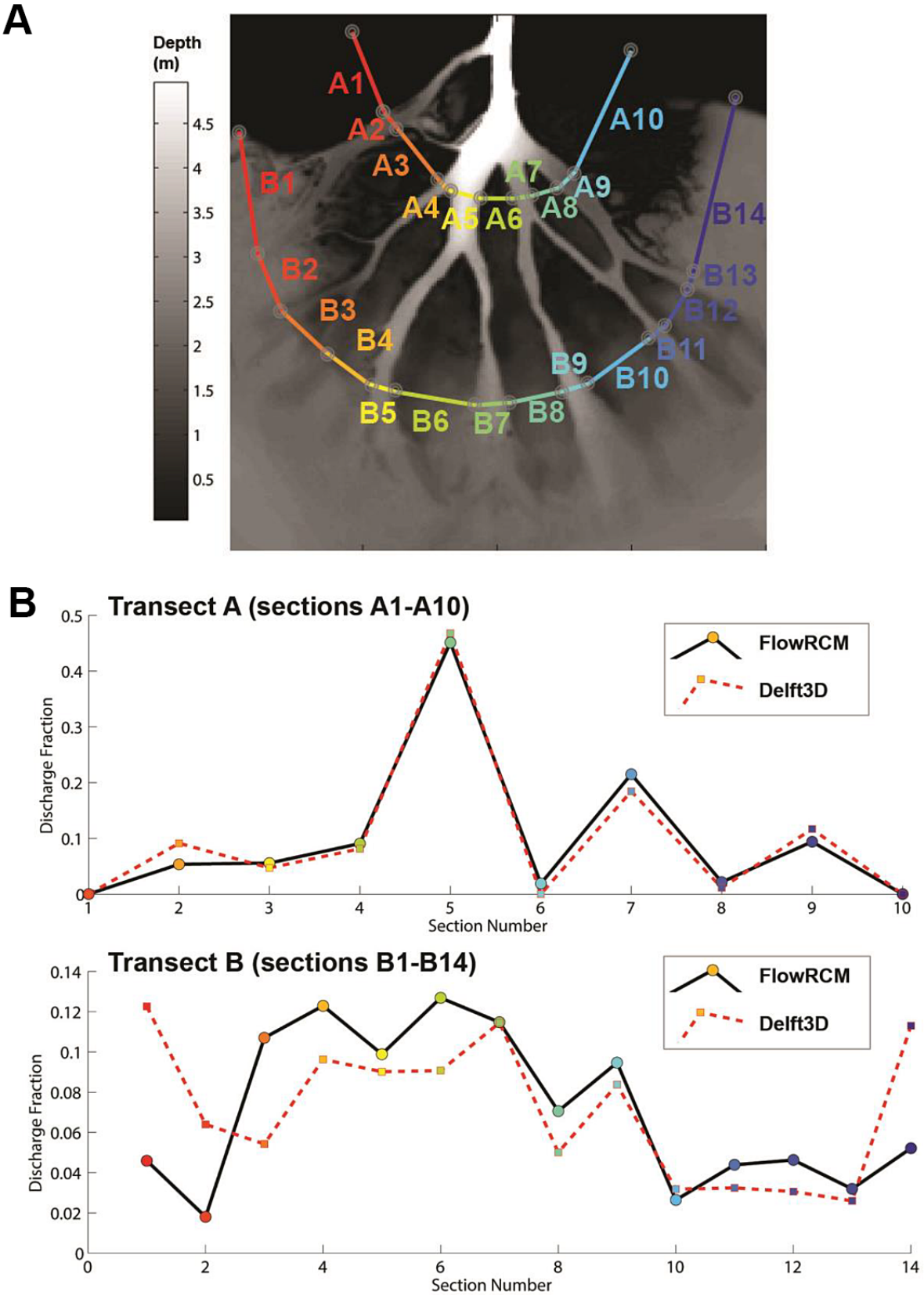

Figure 15. Spatial distribution of water discharge at two transects on the synthetic WLD topography. (a) Transect A is divided into 10 sections and transect B is divided into 14 sections. The fraction of discharge at each section is calculated by the discharge across that section normalized by the total discharge across the whole transect. (b) Results of FlowRCM and Delft3D are plotted for each transect.

cate (i) the nonlinear response of flow velocity to the height of the submerged bump structure, (ii) the discharge partitioning between two competing bifurcation branches in a single bifurcation, and (iii) the discharge partitioning through a whole distributary network of channels and floodplains. The results also show that FlowRCM falls short with respect to Delft3D in terms of detailed hydrodynamic features. For example, in the single bifurcation test, FlowRCM results show strong "local effects" resulting into the flow splitting right in front of the island. Also, occasional irregularities appear due to the probabilistic approach used for routing water parcels.

Overall, FlowRCM performs well at the multichannel network scale, where spatial flux distribution matters more than detailed channel flow structure. Furthermore, for singlechannel-scale cases, such as submerged smooth obstacles, FlowRCM is able to predict regions of high and low velocities, and regions of water surface superelevation and drawdown, although the exact shape of the regions or the values of 
A

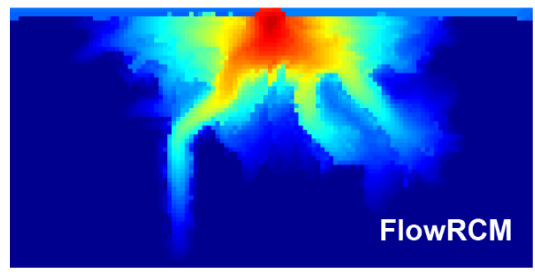

Water surface elevation

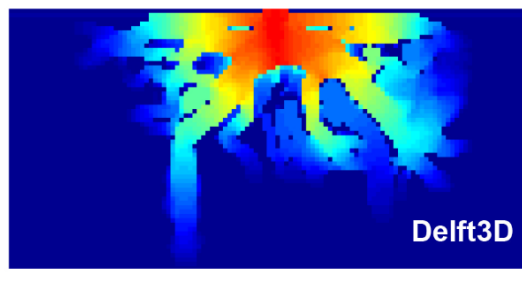

B

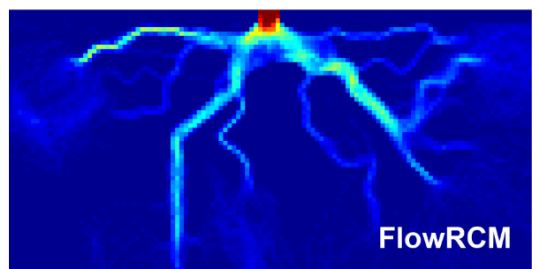

Water unit discharge

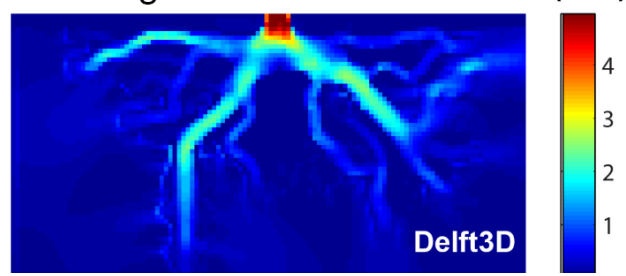

(m)

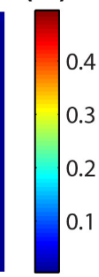

$\left(\mathrm{m}^{2} / \mathrm{s}\right)$

Figure 16. Results of test $4 \mathrm{~b}$ (flow over a DeltaRCM-generated delta topography): (a) water surface elevation and (b) water unit discharge. The results obtained with DeltaRCM are compared to the results from Delft3D using the same topography and input conditions. The results show that (1) both models yield similar discharge distribution throughout the topography, and (2) both models yield similar water surface profiles in magnitude, although DeltaRCM shows a more consistent surface profile and more pronounced gradient across the channel into the floodplains.

the velocity and water surface elevation may be more qualitatively rather than quantitatively correct.

While Flow RCM has been mainly applied in low-gradient environments where flow is typically subcritical, the routing method can be applied to high-gradient and supercritical systems, where topographic slope itself is a reasonable representation of water surface slope and is sufficient for routing fluxes. The laboratory-scale alluvial fan simulation in Part 1 is an example of the application of FlowRCM to high-gradient environments. The key parameters for switching between these environments are the slope/inertia partitioning parameter $\gamma$ for calculating routing probabilities and the 1-D profile equation for calculating the water surface elevation.

One characteristic of rule-based RCMs is the flexibility in making changes to one specific rule in the model, resulting in the opportunity to isolate processes, understand their effects and the behavior of the model, and potentially guide future model improvements. For example, FlowRCM does not resolve the water surface profile based on 2-D hydrodynamic equations. The water surface plays a role in distributing water fluxes through a feedback mechanism: the convergence of water flux causes water surface to rise up along the flow path, introducing a positive surface gradient pointing away from the cells with high flux and diverting flux sideways. This process is done iteratively and the delay in the responses between water surface and water flux causes an oscillation in the model outputs (example from test $4 \mathrm{a}$ in Fig. 13). At the same time, this iterative feedback mechanism allows the water flux to adjust beyond the conditions of the immediate neighbors, thus reducing local effects. A similar iterative feedback mechanism can be found in the row-by-row depthbased iteration method developed by Nicholas (2010).

FlowRCM does have dependency on grid resolution, as each cell only sees its immediate neighbors and the flux is routed using cells as units rather than physical distances. This is widely considered a fundamental property of cellular approaches (Nicholas, 2005), and has been shown in a number of cellular routing models (e.g., Doeschl-Wilson and Ashmore, 2005). The dependency on grid resolution could potentially be removed by introducing a relationship between RC model process parameterization and spatial resolution (Nicholas et al., 2012).

The goal of FlowRCM is not to achieve an accurate solution of the water surface elevation, but to reproduce morphodynamic features obtained so far only by high-fidelity models. As discussed in the companion paper (Liang et al., 2015), the inclusion of the water surface calculation is required but its accuracy can be relaxed when modeling deltas. While our work addresses the plausibility of the model's hydrodynamic results, it is interesting to investigate whether the ability of a flow routing scheme to correctly predict hydrodynamics controls its ability to produce plausible morphodynamic features. We can think, for example, about the process of bifurcation formation. In DeltaRCM a channel mouth goes through a series of stages (Fig. 17): (i) flow expands and sediment deposits with reduced flow velocity, which creates a wide lunate bar with noisy surface; (ii) the irregular topography of the bar forms "ridges" and "troughs" where troughs attract more water flux than ridges; (iii) the enhanced flow rate in troughs prevents sediment from settling while ridges continue to experience deposition; and (iv) the pro- 
Conceptual sketch of channel mouth cross-section over time

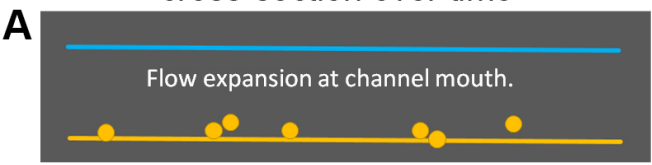

Sediment deposits at channel mouth.

B

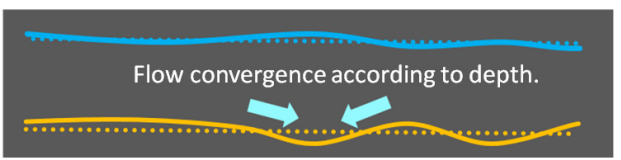

Troughs and ridges develop.

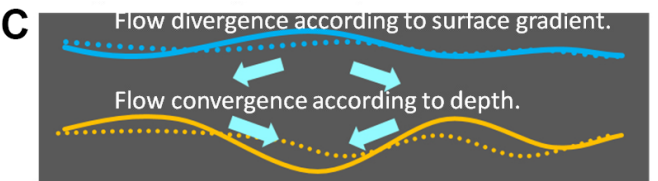

Some troughts become established channels and the ridges on the side become levees.
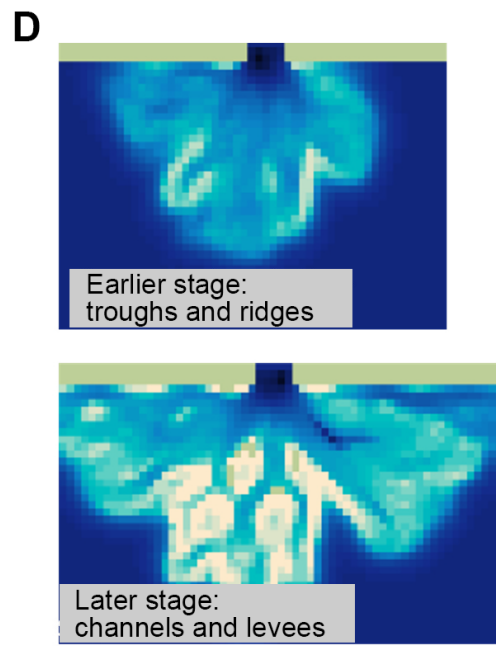

Figure 17. Illustration of the mechanics of bifurcation formation in the DeltaRCM: (a) flow expands and sediment deposits with reduced flow velocity, which creates a wide lunate bar with noisy surface; (b) the irregular topography of the bar forms "ridges" and "troughs" where troughs attract more water flux than ridges, the enhanced flow rate in troughs prevents sediment from settling while ridges continue to experience deposition; (c) the process reaches equilibrium by a feedback mechanism between water surface elevation and water flux. The few paths established from the troughs outcompete the others. (d) A sample simulation from DeltaRCM shows two stages of the conceptual processes described above: the earlier stage with troughs and ridges, and the later stage with channels and levees.

cess reaches equilibrium through the feedback mechanism between water surface elevation and water flux. The few paths established from the troughs outcompete the others. Interestingly, this process not only produces bifurcations, but also trifurcations. Accordingly, DeltaRCM suggests that distributary channel networks are not necessarily solely built by mouth-bar-induced flow bifurcation (Edmonds and Slingerland, 2007; Mariotti et al., 2013).

Another example of the translation from modeled hydrodynamic features to modeled morphodynamic features is the sensitivity of cellular flow routing to topographic details, such as flow acceleration and changes in flow orientation over short distances (Nicholas et al., 2012), which are caused by local effects. In test $4 a$, the results from Delft3D look more "diffusive" than FlowRCM. Local effects, when combined with the Lagrangian representation of fluxes as parcels, may offer a richer flow pattern in response to small changes on the floodplain. Although this observation requires further investigation, based on our results RC flow routing schemes seem to be less constrained by complex flow boundaries caused by wet-dry partitioning than higher-fidelity models based on partial differential equations.

CFD has the advantage of being able to resolve 3-D details such as velocity gradients normal to the bed and turbulence structures. The need for computational efficiency, uncertainty in initial and boundary conditions and lack of detailed information on parameterization for direct simulation often prompt the use of simpler modeling approaches. For example, depth-averaged models with appropriate parame- terizations of 3-D features (e.g., spiral flow in bends) can be used for realistic sediment transport modeling (e.g., Falcini and Jerolmack, 2010). Simple rule-based RCMs are able to resolve hydrodynamic details previously believed to be produced by CFD exclusively (Wolfram, 2002). Such models share many common characteristics as the lattice Boltzmann method for fluid flow (which is considered a class of CFD). As for sediment transport, the detailed 3-D structure flow may be important to many morphodynamic features, which cannot be captured by simple depth-averaged flow routing methods. Identifying these delicate linkages between hydrodynamics and morphodynamics requires the combined effort of CFD modelers and RC modelers.

FlowRCM shares key features with existing RC flow routing schemes (Bates et al., 2010; Nicholas et al., 2012; Pelletier, 2008; Larson and Harvey, 2011), but differs in several ways. First, in order to model low-gradient environments, flow routing should be based on water surface slope, rather than on topographic slope. Some models assume a constant slope throughout the calculation region (Larson and Harvey, 2011) and others almost reproduce the exact solution of water surface profiles in benchmark cases (Bates et al., 2010). FlowRCM calculates the water surface elevation with the purpose of determining the direction of the water flux, without aiming at an exact solution. The underrelaxation of this condition helps us understand to what extent large-scale flow partitioning in deltaic environment is sensitive to hydrodynamic details. Second, FlowRCM does not require predefined maximum flow depth or minimum slope 
for its iterations as in successive MFD iterations (Pelletier, 2008). Third, FlowRCM uses a Lagrangian approach to route fluxes. The routing direction is calculated iteratively, eliminating the need of the traditional "row-by-row" dischargesplitting method as in Nicholas et al. (2012), while handling the multidirectionality typical of distributary networks. An intercomparison between flow routing schemes in a deltaic environment will be the subject of future research.

\section{Conclusions}

In this work we have applied a series of numerical tests to validate the reduced-complexity flow routing scheme, FlowRCM, which is introduced in Part 1 (Liang et al., 2015) as the hydrodynamic component of our RC delta formation model. We selected key hydrodynamic processes essential to channel processes in deltas and designed numerical tests as "benchmarking" cases for this specific environment. We compared the output from FlowRCM with the output from a higher-fidelity hydrodynamic model, Delft3D, which is based on rigorous CFD solutions. We also used theoretical solutions to demonstrate FlowRCM's ability to reproduce hydrodynamic details.

The results show that FlowRCM is able to reproduce most of the flow and water surface features of interest. Overall, it captures (1) the trend of water surface from upstream to downstream and from channels to floodplain, and (2) flow partitioning corresponding to complex bed topography such as flow divergence and convergence around obstacles. Furthermore, the routing scheme is able to produce morphodynamic features such as mouth bars, bifurcations and levee formation. The responsible process for these morphodynamic features is an instability-feedback mechanism resulting from the coupling of the hydrodynamic component and the sediment transport rules.

This work suggests an assessment framework for RCMs that include explicit flow routing schemes. The key ideas include (1) designing test cases to evaluate the performance of the routing scheme in producing features related to the processes of interest, (2) identifying the effects of model rules and connecting them to model output individually, and (3) connecting the hydrodynamic to the morphodynamic performance to evaluate the flow routing scheme's ability to model morphodynamics accurately.

We suggest that FlowRCM is appropriate for modeling environments with multichannel networks where morphodynamic features can be produced from the estimation of channel-to-channel and channel-to-island/floodplain flow partitioning. More detailed prediction of in-channel flow patterns such as spatial distribution of high/low velocity and surface deformation could also be achieved but FlowRCM has not been designed for small-scale engineering applications.
Acknowledgements. M. Liang acknowledges support from National Science Foundation via the National Center for Earthsurface Dynamics (NCED) under agreements EAR-1246761. The authors also acknowledge support from the National Science Foundation (FESD/OCE-1135427, CAREER/EAR-1350336 and GSS/BCS-1063228 to P. Passalacqua, and OCE-1061380 to D. A. Edmonds). The authors also thank A. Ashton and the anonymous reviewer for their insightful opinions.

Edited by: S. Castelltort

\section{References}

Bates, P. D. and De Roo, A. P. J.: A simple raster-based model for flood inundation simulation, J. Hydrol., 236, 54-77, 2000.

Bates, P. D., Horritt, M. S., and Fewtrell, T. J.: A simple inertial formulation of the shallow water equations for efficient twodimensional flood inundation modelling, J. Hydrol., 387, 33-45, 2010.

Chow, V. T.: Open-channel hydraulics, in: Open-Channel Hydraulics, McGraw-Hill, New York, 1959.

Coulthard, T. J., Macklin, M. G., and Kirkby, M. J.: A cellular model of Holocene upland river basin and alluvial fan evolution, Earth Surf. Proc. Land., 27, 269-288, 2002.

Doeschl-Wilson, A. B. and Ashmore, P. E.: Assessing a numerical cellular braided-stream model with a physical model, Earth Surf. Proc. Land., 30, 519-540, doi:10.1002/esp.1146, 2005.

Duan, J. G. and Julien, P. Y.: Numerical simulation of meandering evolution, J. Hydrol., 391, 34-46, 2010.

Edmonds, D. A. and Slingerland, R. L.: Mechanics of river mouth bar formation: implications for the morphodynamics of delta distributary networks, J. Geophys. Res., 112, F02034, doi:10.1029/2006JF000574, 2007.

Edmonds, D. A. and Slingerland, R. L.: Stability of delta distributary networks and their bifurcations, Water Resour. Res., 44, W09426, doi:10.1029/2008WR006992, 2008.

Edmonds, D. A., Slingerland, R., Best, J., Parsons, D., and Smith, N.: Response of riverdominated delta channel networks to permanent changes in river discharge, Geophys. Res. Lett., 37, L12404, doi:10.1029/2010GL043269, 2010.

Edmonds, D. A., Paola, C., Hoyal, D. C. J. D., and Sheets, B. A.: Quantitative metrics that describe river deltas and their channel networks, J. Geophys. Res., 116, F04022, doi:10.1029/2010JF001955, 2011.

Falcini, F. and Jerolmack, D. J.: A potential vorticity theory for the formation of elongate channels in river deltas and lakes, J. Geophys. Res., 115, F04038, doi:10.1029/2010JF001802, 2010.

Ferguson, R. I., Church, M., and Weatherly, H.: Fluvial aggradation in Vedder River: testing a one-dimensional sedimentation model, Water Resour. Res., 37, 3331-3347, 2001.

Freeman, T. G.: Calculating catchment area with divergent flow based on a rectangular grid, Comput. Geosci., 17, 413-422, 1991.

Hajek, E. A. and Wolinsky, M. A.: Simplified process modeling of river avulsion and alluvial architecture: connecting models and field data, Sediment. Geol., 257, 1-30, 2012.

Hardy, R. J., Lane, S. N., Ferguson, R. I., and Parsons, D. R.: Assessing the credibility of a series of computational fluid dynamic 
simulations of open channel flow, Hydrol. Process., 17, 15391560, 2003

Howard, A. D.: Badland morphology and evolution: interpretation using a simulation model, Earth Surf. Proc. Land., 22, 211-227, 1997.

Jerolmack, D. J. and Paola, C.: Complexity in a cellular model of river avulsion, Geomorphology, 91, 259-270, 2007.

Karssenberg, D. and Bridge, J. S.: A three-dimensional numerical model of sediment transport, erosion and deposition within a network of channel belts, floodplain and hill slope: extrinsic and intrinsic controls on floodplain dynamics and alluvial architecture, Sedimentology, 55, 1717-1745, 2008.

Kleinhans, M. G., Jagers, H. R. A., Mosselman, E., and Sloff, C. $\mathrm{J} .:$ Bifurcation dynamics and avulsion duration in meandering rivers by one-dimensional and three-dimensional models, Water Resour. Res., 44, W08454, doi:10.1029/2007WR005912, 2008.

Kleinhans, M. G., Ferguson, R. I., Lane, S. N., and Hardy, R. J.: Splitting rivers at their seams: bifurcations and avulsion, Earth Surf. Proc. Land., 38, 47-61, 2013.

Lane, S. N., Hardy, R. J., Elliott, L., and Ingham, D. B.: Highresolution numerical modelling of three-dimensional flows over complex river bed topography, Hydrol. Process., 16, 2261-2272, 2002.

Larsen, L. G. and Harvey, J. W.: Modeling of hydroecological feedbacks predicts distinct classes of landscape pattern, process, and restoration potential in shallow aquatic ecosystems, Geomorphology, 126, 279-296, 2011.

Lesser, G. R., Roelvink, J. A., van Kester, J. A. T. M., and Stelling, G. S.: Development and validation of a three-dimensional morphological model, Coast. Eng., 51, 883-915, 2004.

Liang, M., Voller, V. R., and Paola, C.: A reduced-complexity model for river delta formation - Part 1: Modeling deltas with channel dynamics, Earth Surf. Dynam., 3, 67-86, doi:10.5194/esurf-367-2015, 2015.

Mariotti, G., Falcini, F. , Geleynse, N., Guala, M., Sun, T., and Fagherazzi, S.: Sediment eddy diffusivity in meandering turbulent jets: Implications for levee formation at river mouths, J. Geophys. Res.-Earth, 118, 1908-1920, doi:10.1002/jgrf.20134, 2013.

Martin, Y. and Church, M.: Numerical modelling of landscape evolution: geomorphological perspectives, Prog. Phys. Geogr., 28, 317-339, 2004.

Murray, A. B.: Reducing model complexity for explanation and prediction, Geomorphology, 90, 178-191, 2007.

Nicholas, A. P.: Cellular modelling in fluvial geomorphology, Earth Surf. Proc. Land., 30, 645-649, 2005.

Nicholas, A. P.: Reduced-complexity modeling of free bar morphodynamics in alluvial channels, J. Geophys. Res.-Earth, 115, F04021, doi:10.1029/2010JF001774, 2010.

Nicholas, A. P.: Modelling the continuum of river channel patterns, Earth Surf. Proc. Land., 38, 1187-1196, 2013.

Nicholas, A. P., Sandbach, S. D., Ashworth, P. J., Amsler, M. L., Best, J. L., Hardy, R. J., Lane, S. N., Orfeo, O., Parsons, D. R., Reesink, A. J. H., Sambrook Smith, G. H., and Szupiany, R. N.: Modelling hydrodynamics in the Rio Paraná, Argentina: an evaluation and intercomparison of reduced-complexity and physics based models applied to a large sand-bed river, Geomorphology, 169-170, 192-211, 2012.
O'Callaghan, J. F. and Mark, D. M.: The extraction of drainage network from digital elevation data, Comput. Vis. Graph. Image Process., 28, 323-344, 1984.

Oreskes, N., Shrader-Frechette, K., and Belitz, K.: Verification, validation, and confirmation of numerical models in the earth sciences, Science, 263, 641-646, 1994.

Overeem, I., Syvitski, J. P. M., and Hutton, E. W. H.: Threedimensional numerical modeling of deltas, in: River Deltas: Concepts, Models and Examples, edited by: Bhattacharya, J. P. and Giosan, L., SEPM Spec. Publ., 83, SEPM, Tulsa, 13-30, 2005.

Paola, C., Twilley, R. R., Edmonds, D. A., Kim, W., Mohrig, D., Parker, G., Viparelli, E., and Voller, V. R.: Natural processes in delta restoration: application to the Mississippi Delta, Ann. Rev. Mar. Sci., 3, 67-91, 2011.

Parsons, J. A. and Fonstad, M. A.: A cellular automata model of surface water flow, Hydrol. Process., 21, 2189-2195, doi:10.1002/hyp.6587, 2007.

Passalacqua, P., Lanzoni, S., Paola, C., and Rinaldo, A.: Geomorphic signatures of deltaic processes and vegetation: the GangesBrahmaputra-Jamuna case study, J. Geophys. Res.-Earth, 118, 1838-1849, 2013.

Pelletier, J. D.: Quantitative Modeling of Earth Surface Processes, Cambridge U. Press, Cambridge, 2008.

Roberts, H., Adams, R., and Cunningham, R.: Evolution of sanddominant subaerial phase, Atchafalaya Delta, Louisiana, AAPG Bull., 64, 264-279, 1980.

Seybold, H., Andrade Jr., J. S., and Herrmann, H. J.: Modeling river delta formation, P. Natl. Acad. Sci. USA, 104, 16804-16809, 2007.

Shaw, J. B., Mohrig, D., and Whitman, S. K.: The morphology and evolution of channels on the Wax Lake Delta, Louisiana, USA, J. Geophys. Res.-Earth, 118, 1-23, 2013.

Siviglia, A., Stecca, G., Vanzo, D., Zolezzi, G., Toro, E. F., and Tubino, M.: Numerical modelling of two-dimensional morphodynamics with applications to river bars and bifurcations, Adv. Water Resour., 52, 243-260, 2013.

Syvitski, J. P. M., Kettner, A. J., Overeem, I., Hutton, E. W. H., Hannon, M. T., Brakenridge, G. R., Day, J., Vörösmarty, C., Saito, Y., Giosan, L., and Nicholls, R. J.: Sinking deltas due to human activities, Nat. Geosci., 2, 681-686, 2009.

Tucker, G. E. and Bras, R. L.: Hillslope processes, drainage density, and landscape morphology, Water Resour. Res., 34, 2751-2764, 1998.

USACE - US Army Corps of Engineers: Atchafalaya River hydrographic survey, 1998-1999: Old River to Atchafalaya Bay including main channel and distributaries, The District, New Orleans, LA, 1999.

Van De Wiel, M. J., Coulthard, T. J., Macklin, M. G., and Lewin, J.: Embedding reach-scale fluvial dynamics within the CAESAR cellular automaton landscape evolution model. Geomorphology, 90, 283-301, doi:10.1016/j.geomorph.2006.10.024, 2007.

Wellner, R., Beaubouef, R., Van Wagoner, J., Roberts, H., and Sun, T.: Jet-plume depositional bodies - the primary building blocks of Wax Lake delta, Gulf Coast Assoc. Geol. Soc. Trans., 55, 867909, 2005.

Willgoose, G.: A statistic for testing the elevation characteristics of landscape simulation models, J. Geophys. Res.-Sol. Ea., 99, 13987-13996, 1994. 
Wolfram, S.: A new kind of science, Wolfram Media, Champaign, IL, 2002.
Wolinsky, M. A., Edmonds, D. A., Martin, J., and Paola, C.: Delta allometry: growth laws for river deltas, Geophys. Res. Lett., 37, L21403, doi:10.1029/2010GL044592, 2010. 